\title{
Game theory and research data sharing
}

\author{
Pronk, T.E. ${ }^{1}$ \\ ${ }^{1}$ KWR Watercycle Research Institute, P.O. Box 1072, 3430 BB Nieuwegein, the Netherlands \\ Corresponding author's e-mail: tessa.pronk@kwrwater.nl
}

Having research data available for others is one of the measures to ensure transparent, reproducible, and reusable results. While reusing research data has evident benefits for the scientific community as a whole, decisions to archive and share these data are primarily made by individual researchers. Is research data sharing also to their advantage? To tackle this question, we built a model in which there is an explicit cost associated with sharing datasets whereas reusing such sets implies a benefit as time is saved in that case. In our calculations, conflicting interests appear for researchers. Individuals are better off if everyone shares, as time is saved by reusing data. At the same time, each individual is better off omitting the sharing cost and not sharing him/herself. A strong policy or solid incentives such as a (citation-) benefit are needed. Making data available would in that latter case become the most profitable, and therefore stable, strategy. This means researchers would willingly make their datasets available, and arguably in the best possible way to enable reuse. These results were published in https://peerj.com/articles/1242/. In an extra calculation, we focus on quantifying the minimum reuse needed to break even on time costs for sharing, to see if current reuse rates are enough to make a sharing community indeed become more efficient. 